\title{
多层螺旋 CT 在肺结核诊断中的应用价值研究
}

\section{Study on the Application Value of Multislice Spiral CT in the Diagnosis of Pulmonary \\ Tuberculosis}

\section{刘欢}

Huan Liu

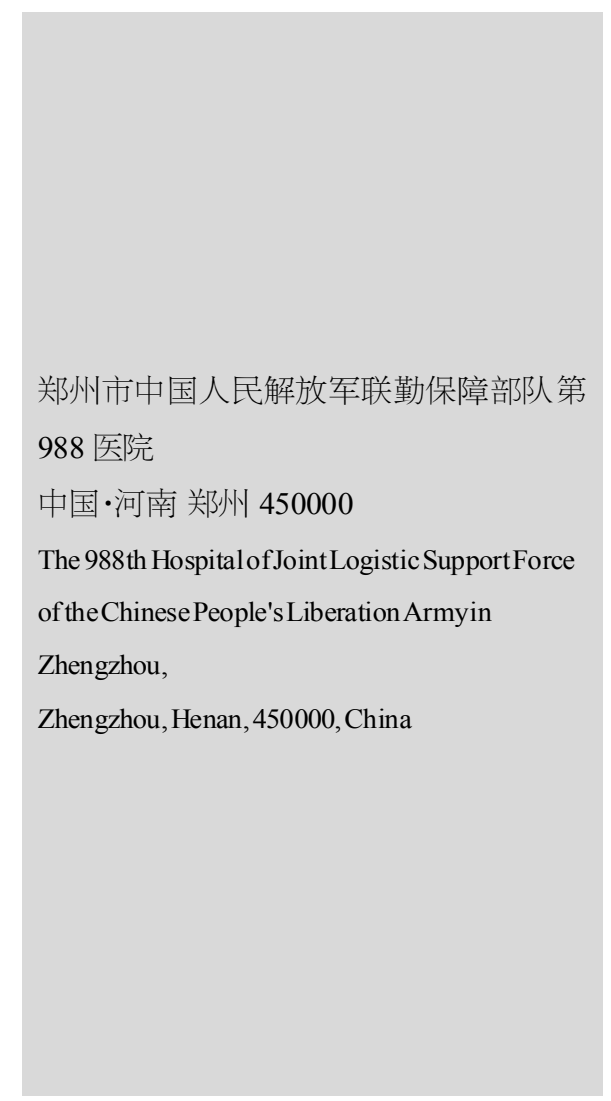

【摘要】论文以采用多层螺旋 CT 技术对肺结核患者进行临床诊断, 并分析该技术的应用 价值为主要研究目的。采用回顾性分析, 通过对本院 2018 年 12 月一 2019 年 6 月收治的 40 例肺结核患者进行诊断,分析多层螺旋 CT 扫描技术在观察肺结核征象方面的诊断价值。由 此得出, 采用多层螺旋 CT 检验存可疑肺结核症状的患者进行诊断, 还能够清楚地对病灶的 具体情况进行观察, 以便通过结核部位、分型、分期的对比来提升临床治疗效果。另外, 通过 多层螺旋 CT 观察纵隔及肺门淋巴结增大情况, 对于观察胸膜肥厚埭连和胸水方面的效果 也更加具备优势, 充分体现了多层螺旋 CT 技术的临床应用价值。

【Abstract \The purpose of this paper is to use multislice spiral CT technology for clinical diagnosis of pulmonary tuberculosis patients and to analyze the application value of this technology. Retrospective analysis was adopted to analyze the diagnostic value of multislice spiral CT scan in observing the signs of tuberculosis through the diagnosis of 40 cases of tuberculosis admitted to our hospital from December 2018 to June 2019. It is concluded that MSCT examination of patients with suspicious pulmonary tuberculosis symptoms can also clearly observe the specific conditions of the lesions, so as to improve the clinical treatment effect by comparing the sites, types and stages of tuberculosis. In addition, the observation of mediastinal and hilar lymph node enlargement by MSCT is more advantageous for the observation of pleural thickening and adhesion and pleural effusion, which fullyreflects the clinical application value ofMSCT.

【关键词】多层螺旋 $\mathrm{CT}$; 肺结核诊断; 应用价值

【Keywords】 multislice spiral CT; tuberculosis diagnosis; application value 【DOI \10.36012/pmr.v1i3.940

\section{1 引言}

肺结核是一种由抗酸结核分枝杆菌繁殖而形成的慢性传 染性疾病, 其在发展过程中累及多个脏器器官, 主要以肺部为 主要部位, 体质虚弱者和老年人较为容易受到感染。当前, 人 们生活方式不断变化,生活和工作压力也逐渐增加,使得许多 人饮食和作息不规律的现象十分明显, 导致身体呈现亚健康 的状态, 给疾病带来了可乘之机, 从而危害人体健康, 给人们 的日常生活带来了负面影响[1]。因此, 及早正确诊断肺结核疾 病, 能够有效提高疾病的治疗效率。CT 检查是肺结核诊断的 主要方法之一, 本文结合本院收治的 40 例肺结核患者作为研
究对象, 分析了他们的多层螺旋 CT 影像, 从而总结和判断多 层螺旋 CT 影像诊断依据及其价值。若肺结核患者未能得到及 时诊治, 很可能致使疾病合并支气管扩张、肺部感染等情况, 导致病情加重, 对患者的生命安全产生威胁。另外, 肺结核属 于传染性疾病, 早诊断、早治疗对于防治疾病传播和维护社会 稳定也具有重要意义。

\section{2 一般资料与方法}

\section{1 一般资料}

本研究采用回顾性分析, 选取本院 2018 年 12 月一-2019 年 6 月经疾检、活检及临床治愈证实为肺结核的 40 例患者作 
为研究对象。其中, 存在男患者 23 例, 女患者 17 例, 患者年龄 分布在 22 74 岁,平均年龄为 $(47.9 \pm 2.4)$ 岁。

\section{2 研究方法}

患者入院后, 均接受胸部平片和薄层 $\mathrm{CT}$ 检查, 并对 20 例 患者进行增强 CT 检查, 具体检测范围包括肺尖到肺底。其中, 检测所应用的设备型号为西门子公司生产的双源 Definition Flash CT, 造影剂使用 $100 \mathrm{~mL}$ 的碘克沙醇注射液对比剂进行 增强扫描,方法为静脉团注, 静脉团注后 $60 \mathrm{~s} 、 120 \mathrm{~s}$ 时, 进行增 强扫描。上述所获得的检查数据, 全部进行 $1 \mathrm{~mm}$ 重建, 并将薄 层重建横断面图像传至工作站, 通过多平面重建的方法对所 获图像进行分析。

\section{3 结果}

在本次研究中, 主要以肺结核的磨玻璃密度和实变、空 洞、随机分布结节和肿块、支气管血管周围间质增厚、树芽征 等征象的检出率为研究内容, 具体结果如下 ${ }^{[2]}$ :

第一, 磨玻璃密度和实变。40 例肺结核患者中, 存在此种 征象的患者共有 22 例 (55.00\%), 具体表现为病灶的分布呈现 不均匀状态, 且密度不等, 存在大小不一的斑块状影像。其中, 有 10 例患者的病灶位于右肺上叶, 4 例患者病灶位于右肺中 叶, 6 例患者病灶位于左肺上叶尖, 2 例患者病灶位于叶间隙, 并能够在 CT 影像中明显观察到支气管气象。

第二,空洞。40 例肺结核患者中, 10 例患者存在空洞的征 象,所占比例为 $25 \%$ 。空洞是指肺结核患者在进行 CT 检查的 一种征象, 在本研究中, 有 5 例患者的空洞征象位于右肺上 叶, 有 3 例患者的空洞征象位于左肺上叶, 1 例患者的空洞征 象位于中叶, 1 例患者位于左肺下叶背段。其中, 有 2 例患者呈 现中等壁厚偏空洞。

第三,随机分布结节和肿块。40 例患者中, 存在随机分布 结节和肿块型的患者共有 6 例 (15\%), 具体表现为密度不等 的圆形及椭圆形, 并且边缘存在明显的毛刺征。其中, 有 3 例 患者的结节和肿块位于右肺上叶, 1 例位于中叶, 2 例位于左 肺上叶。

第四, 树芽征。存在树芽征的患者有 20 例 ( 50\%)。第五, 支气管、血管周围间质增厚。存在体力患者具备支气管、血管 周围间质增厚的征象, 其所占比例为 $(17.5 \%)$ 。另外,通过增强 扫描的 20 例患者, 有 5 例患者未呈现明显强化特征, 7 例患者 呈现轻度强化,9 例患者呈现中度强化。

\section{4 讨论}

结核杆菌进入患者肺部并引发的一系列脏器衰损的慢性 感染疾病被称为肺结核，其具备一定的传染性特征。一般来 说, 肺结核的主要特点为由于病灶干酪样坏死并发生钙化和 纤维化，所以通常利用痰检无法及时诊断患者病情。通过相关 统计数据表明，利用痰检方法来诊断肺结核的准确性通常低 于 50\%。随着科技的不断发展, 当前主要通过影像学检查来实 现肺结核的诊断。

多层螺旋 CT 检查的优势在于无创口、操作简便等, 在医 学影像检查中得到了广泛应用。通过多层螺旋 CT 检查, 能够 显著检查到肺结核磨玻璃密度和实变、空洞、随机分布的结节 与肿块、支气管血管周围间质增厚、树芽征、胸水、肺内球形结 合等征象 ${ }^{[3]}$ 。该技术的应用, 能够有效减少肺结核疾病诊断过 程中的误诊、漏诊现象, 提高诊断准确性, 对于帮助医师合理 制定治疗方案, 促进患者早日恢复健康具有积极意义。

在此以部分肺结核征象为例,分析其临床诊断特征。磨玻 璃密度和实变主要于肺炎疾病、肺部肿瘤和支气管感染等方 面可见，在临床上容易将细菌性肺炎或支原体肺炎和肺结核 相混淆, 从而导致误诊, 因此在具体诊断过程中需要加以重 视。肺结核的空洞征象, 在多层螺旋 CT 检查中主要表现为薄 壁空洞和厚壁空洞，两种类型的空洞能够反映不同时期的肺 部病理变化。

\section{5 结语}

综上所述，采用多层螺旋 CT 技术获取肺结核的医学影 像, 能够观察到多样化的肺结核征象, 从而明确判别患者肺结 核的结核部位、分型等情况,便于医师进一步制定肺结核治疗 方案, 具有重要的临床应用价值。因此, 多层螺旋 CT 检查肺结 核疾病, 能够有效提升肺结核的检出率, 清楚地显示出肺结核 病灶的边缘、内部及各种征象, 对提升患者康复率具有积极意 义。

\section{参考文献}

[1]吴平洋.多层 CT 在肺结核诊断中的应用价值分析[J].基层医学 论坛,2019,23(23):3357-3358.

[2]买尔哈巴・衣马木,吐尔洪・艾则孜.多层螺旋 CT 在肺结核诊断 和鉴别诊断中的应用价值[J].影像研究与医学应用,2019,3(12):221-222.

[3]刘业辉.多层螺旋 CT 扫描对于肺结核诊断的应用价值分析 [ J] 影像研究与医学应用,2018,2(24):72-73. 